\section{P3.91 ANTIRETROVIRAL THERAPY DISCONTINUATION AMONG HIV INFECTED ADULTS IN ETHIOPIA IN 2003-2015: PREVALENCE, TREND AND RISK FACTORS}

${ }^{1}$ Hailay Gesesew, ${ }^{2}$ Prof Paul Ward, ${ }^{1}$ Prof Kifle Hajito, ${ }^{2}$ Lillian Mwanri. ${ }^{1}$ Jimma University, Jimma, Ethiopia; ${ }^{2}$ Flinders University, Adelaide, Australia

\subsection{6/sextrans-2017-053264.326}

Introduction Antiretroviral therapy (ART) discontinuation obscures achievements for the UNAIDS treatment targets 2 and 3. Nevertheless, the magnitude, trend and its risk factors are not explored contextually. We carried out historical data analysis to assess prevalence, trend and risk factors for ART discontinuation among adults in Southwest Ethiopia.

Methods 12 years retrospective cohort analysis was performed with 4900 HIV-infected adults between 21 June 2003 and 15 March 2015 registered at the ART clinic at Jimma University Teaching Hospital. ART Discontinuation could be lost to follow-up, defaulting and/or stopping medication while remaining in care. 10 years trends for ART discontinuation was described using a line graph. We used binary logistic regression to identify factors that were correlated with ART discontinuation. To handle missing data, we applied multiple imputations assuming missing at random pattern.

Results In total, 4900 adults enrolled on ART, of whom 1090 (22.4\%) had discontinued, 954 (19.6\%) had transferred out, $300(6.2 \%)$ had died, and the remaining 2517 (51.8\%) were alive and on ART between 2003 and 2015. The recent trend of ART discontinuation showed an upward direction reaching a peak in 2004 and 2005 with 10\%. Being female (AOR $=2.1,95 \% \mathrm{CI}: 1.7-2.8$ ), having an immunological failure (AOR $=2.3$, 1.9-8.2), having tuberculosis/HIV co-infection $(\mathrm{AOR}=1.5,1.1-2.1)$ and no previous history of HIV testing $(\mathrm{AOR}=1.8$, 1.4-2.9) were the risk factors for ART discontinuation.

Conclusion One of five adults had discontinued from ART, and the trend of ART discontinuation increased recently. Discontinued adults were more likely to be females, tuberculosis/ HIV co-infected, with immunological failure and no history of HIV testing. Therefore, it is vital to implement effective programs such as community ART distribution and linkage-casemanagement to enhance ART linkage and retention.

\section{P3.92 THE CASE OF THE GONORRHOEA SECRET SHOPPER: A REVIEW OF ONLINE PRESCRIBING PRACTICES, ENGLAND, 2016}

Hamish Mohammed, Helen Fifer, Gwenda Hughes. Public Health England, London, UK

10.1136/sextrans-2017-053264.327

Introduction The number of private online sexually transmitted infection testing and treatment providers in England is increasing. While over $90 \%$ of gonorrhoea cases seen at sentinel specialist sexual health clinics in England are prescribed the recommended dual therapy of ceftriaxone (intramuscular) and azithromycin (oral), the extent to which private online providers adhere to national treatment guidelines is unclear. We systematically reviewed online search results to assess the antibiotic courses prescribed for gonorrhoea treatment.

Methods A Google search for 'gonorrhoea treatment online' was performed using a private internet browsing session of Chrome in November 2016. The first 2 pages of search results were reviewed to assess whether they were links to gonorrhoea treatment providers and, if so, which antibiotic course was available for purchase. A thematic analysis of the medical advice given online was also performed, and frequencies and proportions are reported.

Results There were 27 unique results on the first 2 pages of search results, 17 (63\%) of which were to online providers. Among these, upon clicking a checkbox to indicate having been diagnosed with gonorrhoea, 71\% (12/17) provided a course of antibiotics for treatment. However, only 1 of these required visiting a terrestrial pharmacy for the recommended dual therapy; the remainder (11/12) provided cefixime and azithromycin tablets for gonorrhoea treatment via next-day delivery. On their websites, online providers widely acknowledged that 'an injection' [of ceftriaxone] is more effective for gonorrhoea treatment, but suggested that it was 'acceptable' to prescribe azithromycin and cefixime.

Conclusion Non-recommended antibiotic courses for the treatment for gonorrhoea are easily accessible online. There is a need to raise awareness among online providers and the public of the recommended dual therapy for gonorrhoea. To prevent the further emergence of antibiotic resistance in Neisseria gonorrhoeae, adherence to the recommended dual therapy in all settings is essential.

\section{P3.93 PREVALENCE OF BACTERIAL VAGINOSIS IN WOMEN PRESENTING RECURRENT VAGINAL DISCHARGE IN MOROCCO}

Hancali Amina, Bellaji Bahija, Jennane Sanae. Nationa Institut of Hygiene- Ministry of Health-Morocco, Rabat, Morocco

\subsection{6/sextrans-2017-053264.328}

Introduction Bacterial vaginosis (BV) is the most common cause of vaginal discharge in women of reproductive age throughout the world. In Morocco, the women consulting for vaginal discharge are systematically treated by the syndromic approach. This study's goal is to investigate this infection in Moroccan women presenting a recurrent discharge even after treatment.

Methods Retrospective study carried out by detailed analysis of case records in the STIs laboratory in the National Institute of Hygiene for a period of 4 years, between January 2010 and December 2015. 2402 female's patients presenting a vaginal discharge were received in the laboratory for the vaginal fluid collections and analysis. Among these women, 305 were pregnant. Cultures were performed for fungal microorganisms. BV diagnosis was based on the presence of clue cells, $\mathrm{pH}>4.5$, and absence of Lactobacilli. Trichomonas vaginalis (TV) identification was performed by culture and by the wet preparation microscopy.

Results All Women received are married and sexually active. The median of age was 34 years (18-50 years). Among the 2402 women registered, 17.7\% had BV, 42\% had Candida and $4.4 \%$ had TV infection. Among the 305 pregnant women, 6.5\% had BV, 38.7\% had Candida and 1\% had TV infection. No infection with Neisseria gonorrhoeae was found in all the women received. In most of cases, strong vaginal discharges with a fishy smell were linked to BV.

Conclusion Our results revealed that the infection due to the candida is the most common cause of the vaginal discharge followed by BV and the TV in both pregnant and sexually active women. In Morocco, even if the BV is not the first 
aetiology that causes vaginal discharge but due of the complications that a recurrent BV infection can cause in women especially pregnant women, its management must be effective in gynecologic and obstetric services and also in the campaigns against STIs and HIV that are regularly conducted at the national level.

\section{P3.94 DOES MASS DRUG ADMINISTRATION WITH AZITHROMYCIN FOR TRACHOMA CONTROL HAVE AN IMPACT ON THE PREVALENCE AND MACROLIDE RESISTANCE OF GENITAL MYCOPLASMA GENITALIUM INFECTION?}

M Harrison, ${ }^{1} \mathrm{M}$ Marks, ${ }^{2} E$ Harding-Esch, ${ }^{3,1} \mathrm{MJ}$ Pond, ${ }^{1} \mathrm{R}$ Butcher, ${ }^{2} \mathrm{~A}$ Solomon, ${ }^{2} \mathrm{NK}$ Tan, ${ }^{4} \mathrm{~A}$ Nori, ${ }^{1} \mathrm{H}$ Kako, ${ }^{5} \mathrm{D}$ Mabey, ${ }^{2} \mathrm{ST}$ Sadiq, ${ }^{1,2,4}$. ' Applied Diagnostic Research and Evaluation Unit, Institute of Infection and Immunity, St George's, University of London, UK; ${ }^{2}$ London School of Hygiene and Tropical Medicine, UK; ${ }^{3}$ HIVISTI Department, Public Health UK; ${ }^{4}$ St George's University Hospitals NHS Foundation Trust; ${ }^{5}$ Ministry of Health and Medical Services, Solomon Islands

\subsection{6/sextrans-2017-053264.329}

Introduction The first round of Mass Drug Administration (MDA) with $1 \mathrm{~g}$ oral azithromycin for ocular Chlamydia trachomatis (CT) infection, which is a key component of trachoma control strategies, concomitantly reduced genital CT infection in the Solomon Islands. However, this dose is known to be sub-optimal for the treatment of genital Mycoplasma genitalium (MG) infection and may also encourage emergence of antimicrobial resistance (AMR) to macrolides in MG.

Methods Pre-MDA and 6 months post-MDA CT-negative selfcollected vulvo-vaginal swabs from women attending three outpatient antenatal clinics (Honiara, Solomon Islands), already investigated for the impact of MDA on genital CT prevalence, were tested for MG infection using nucleic acid amplification. MG positive samples were subsequently tested for macrolide resistance by sequencing domain $\mathrm{V}$ of $23 \mathrm{~S}$ rRNA DNA region of MG.

Results MG positivity was found in $11.9 \%$ (28/236) of women pre-MDA and in $10.9 \% \quad(28 / 256) 6$ months post-MDA $(p=0.7467) .22 \mathrm{MG}$ positives from each of the pre-MDA and post-MDA samples were sequenced, all showing a macrolide susceptible genotype.

Conclusion A single MDA round in an island population with apparent high MG prevalence with $1 \mathrm{~g}$ azithromycin did not impact on either MG positivity or detection of genetically determined macrolide resistance in this population, in contrast to decreased genital CT positivity in the same population. It is unclear if this apparent lack of impact is due to inadequate efficacy of single-dose azithromycin or transmission dynamics of the infection. Further investigation of the impact of multiple rounds of MDA on antibiotic-experienced and -naïve populations is warranted.

\section{P3.95 QUALITY OF CARE AND INCIDENCE OF STIS IN A COHORT OF TRANSGENDER WOMEN LIVING WITH HIV}

HEL Reno, R Presti, T Brown, BP Stoner. Division of Infectious Disease, Washington University in St. Louis, MO, USA

10.1136/sextrans-2017-053264.330

Introduction Transgender women are at high risk for HIV infection, but less is understood about their rates of sexually transmitted infections (STIs) and the quality of sexual health services they receive. The Washington University Virology Clinic (St. Louis, MO USA) serves patients living with HIV; $2 \%$ of patients report being transgender women.

Methods We conducted a retrospective cohort study of transgender women living with HIV $(n=41)$ to document frequency of testing for incident bacterial STIs [syphilis, gonorrhoea (GC), chlamydia (Ct)], with demographic information, markers of HIV care, and STI test results from 20112015.

Results Most patients were African American (91\%) and on antiretroviral medications (>88\%), although only $56 \%$ maintained HIV viral load suppression. Health challenges included a history of disrupted antiretroviral treatment $(66 \%)$ and STIs $(65 \%)$. Incident syphilis was diagnosed in $7.3 \%$ patients, and GC and Ct were diagnosed in $19.5 \%$ and $9.8 \%$ of patients, respectively. For syphilis screening, $90 \%$ of patients were tested at least once a year, and $53 \%$ of patients were tested more than once a year. For GC/Ct screening, $89 \%$ of patients were tested at least once a year and $49.3 \%$ of patients were tested more than once a year. For patients with GC or $\mathrm{Ct}$ infection, only $44 \%$ of patients were retested at the site of infection 3-6 months after treatment. Reinfection with GC or $\mathrm{Ct}$ was subsequently diagnosed in $19.5 \%$ of patients. Frequency of three site testing for GC/Ct (genital, rectal, pharyngeal) increased over the course of the study period (from $3 \%$ of patients to $34 \%$ of patients, $\left.\mathrm{X}^{2}=17.69, \mathrm{p}=0.001\right)$.

Conclusion Transgender women living with HIV are at high risk for incident bacterial STIs. Frequency of testing for STIs increased over a five-year period, but many patients with documented infection were not re-tested after treatment as recommended by current guidelines. Understanding STI rates, primary locations of infection, and lack of retesting in patients will improve patient education and standardise care for patients.

\section{P3.96 FACTORS ASSOCIATED WITH COMMERCIAL SEXUAL BEHAVIOUR AMONG MEN WHO HAVE SEX WITH MEN IN SHENZHEN, 2011 TO 2015}

${ }^{1}$ Hong Fuchang, ${ }^{1}$ Cai Yumao, 'Lai Yonghui, 'Wen Lizhang, ${ }^{1} Y u$ Weiye, ${ }^{2}$ Liu Hui. ${ }^{1}$ Shenzhen Centre for Chronic Disease Control and Prevention, Shenzhen, China; ${ }^{2}$ National Centre for AIDS/STD Control and Prevention, China CDC

\subsection{6/sextrans-2017-053264.331}

Introduction With the use of new-type drugs, Money boys (MB) will have unprotected sex and injection behaviours easily, thus accelerate the spread of HIV among men who have sex with men(MSM) through sexual transmission and blood transmission. Our study is to investigate the status and factors associated with commercial sexual behaviour among MSM in Shenzhen, China.

Methods Convenience sampling method was used to recruit MSM from 2011 to 2015. Questionnaire-based interviews were conducted on a one-on-one basis. Data were collected including socio-demographic information, HIV testing history, history of blood donation and drug abuse in recent two years, self-identified sexual orientation, role in homosexual behaviour, ever being $\mathrm{MB}$ and clients of MB. $5 \mathrm{ml}$ blood samples were taken and tested for treponema pallidum and HIV antibodies.

Results Among the total of 3040 MSM recruited,341 (11.2\%) reported ever being MB. The prevalence of syphilis, HIV, and 